\title{
Quantum gravity as a metaphysical problem
}

\begin{abstract}
The problem with quantum gravity is usually presented as if it would be difficult to construct even a single quantum theory of relativistic gravity. This is shown to be wrong. A straightforward approach using standard, well-studied methods allows to construct mathematically well-defined quantum theories which give, in a certain classical limit, the Einstein equations of GR: GR may be transformed into a field theory on a fixed background by breaking diffeomorphism symmetry using harmonic coordinates. The resulting field theory may be regularized using standard lattice approximation techniques. The result is a well-defined canonical theory with a finite number of degrees of freedom, which can be quantized without problems in a canonical way.
\end{abstract}

Why such a straightforward way to quantize gravity is simply ignored? We identify missing explanation of relativistic symmetry as an important argument, and propose a solution. evaluate possible explanations why this simple possibility to construct a theory of quantum gravity is ignored. While a lot of different metaphysical and sociological reasons play a role, we identify as a main point a preference of the scientific community for the relational philosophy behind the spacetime interpretation of GR, in opposition to the Newtonian concept of absolute space and time (substantivalism).

We conclude that the quantization of gravity is not a problem of physics, but a metaphysical problem. It is a problem of the relational philosophy of space and time in the tradition of Descartes and Leibniz, which is the base of the spacetime interpretation of GR, because this philosophy is incompatible with the known examples of theories of quantum gravity.
Volume I Issue 5 - 2017

\section{Ilja Schmelzer}

Independent Researcher with expertise in Theoretical Physics, Elementary Particle Physics, Germany

Correspondence: Ilja Schmelzer, Independent Researcher with expertise in Theoretical Physics, Quantum Physics, Elementary Particle Physics, Germany, Tel 4930631625I, Email ilja.schmelzer@gmail.com

Received: September 01, 2017 | Published: November 29, 2017

\section{Introduction}

Quantum gravity is usually represented as being one of the central open problems of modern fundamental physics. It is presented as being "a dream, a theoretical need and a scientific goal. It is a theory which still does not exist in complete form", a "yet-to-be-found theory". ${ }^{1}$ The starting point of this paper is that such a non-existence claim is wrong: There exists a simple and straightforward way to construct a quantum theory of gravity-that means, a well-defined quantum theory, which in some well-defined limit gives the classical Einstein equations of GR. Moreover, the technical way how to do this is well-known and wellunderstood in all of its parts.

We start with the description of this straightforward construction of a theory of quantum gravity. After this, we discuss the metaphysics of quantum gravity. We find that missing explanation of relativistic symmetry would be the most important metaphysical objection to straightforward QG. But this problem appears solvable. Another important argument proposed by Rovelli is instead, nothing but a metaphysical preference in the age-old philosophical dispute between relational and absolute theories to space and time. And given that quantum gravity on an absolute background exists, but a relational, background-independent quantum gravity doesn't, this is not an argument against quantum gravity with a background, but an argument against relationalism.

We also discuss, shortly, various historical, psychological and sociological influences which distort the actual metaphysical discussion.

\section{How GR can be quantized in a simple way}

The first step is known as the field-theoretic approach to GR. As a starting reference, one can use Feynman's comments at the Chapel Hill conference $1957 .^{2}$ The key idea is to consider the gravitational field as a field on some affine background spacetime. For weak gravitational fields, one would say consider fluctuations of the gravitational field $g_{\mu \nu}(x)$ around the vacuum solution, the Minkowski metric $\varepsilon_{\mu \nu}$. The metric $g_{\mu v}$ is then, "just a shorthand for $h_{\mu v}(x)$ ", with $h_{\mu v}(x)$ being the gravitational field. Feynman says: "Obviously one loses the beauty of geometry but this is not primary. What is primary is that one had a new field and tried his very best to get a spin-two field as consistent as possible". Feynman was aware of general covariance as a symmetry of these equations, and comments: "From the other viewpoint the geometry is important, but from this viewpoint gravity is just another field. I am sure that an enormous amount of formulae would be collected without having the generally covariant quantum theory".

Let's note that using GR as a field theory is clearly already a modification of GR, because it already fixes the topology of the spacetime to be $\mathbb{R}^{4}$. But quantum gravity is not obliged to preserve GR in all details. Given that we have not yet observed any GR solutions with non-trivial topology, a restriction of the theory to is viable, and this is., all one needs to preserve the empirical success of GR is a theory which, in certain limit, gives the Einstein equations of GR. 


\section{How to handle diffeomorphism symmetry}

Symmetries are in general beautiful, but they can cause serious problems for quantization. This is the case for the quantization of GR, because GR has a very large symmetry group - the group of diffeomorphisms. In the field-theoretic approach to gravity this diffeomorphism symmetry of GR has to be broken. It is essentially broken already by the formula $g_{\mu v}(x)=\varepsilon_{\mu v}+h_{\mu v}(x)$, which introduces a difference between the vacuum solution $g_{\mu \nu}(x) d x^{\mu} d x^{\nu}$ and another, non-trivial solution $g_{\mu \nu}(x) d x^{\mu} d x^{v}$. Make a coordinate transformation $x^{\prime}=x^{\prime}(x)$. This does not change the metric-it remains the same metric, so, the same solution from point of view of GR. But $g_{\mu^{\prime} v^{\prime}}\left(x^{\prime}(x)\right) d x^{\mu^{\prime}} d x^{\nu^{\prime}}$ will be some completely different field $g_{\mu \nu}^{\prime}(x) d x^{\mu} d x^{v}$, thus, define some completely different function $h_{\mu v}(x)$. So, "the same" solution of GR has in the field-theoretic approach many different realizations.

This situation is well-known from gauge theory, where we have also a quite large group of continuous symmetries, gauge transformations. And there have been developed methods to handle this, so that one can, nonetheless, use standard field theory. One standard approach proposed by Faddeev and Popov uses a gauge condition to choose one field configuration out of each equivalence class, does all the computations using the field theory with this gauge condition as an additional equation, and then corrects for the error which is nonetheless made: The abstract space of solutions one would obtain by factorizing is nonetheless different than its embedding, with a gauge condition, into the field theory. The correction is defined by a so-called "Faddeev-Popov ghost". According to Donoghue, this works nicely for GR as an effective field theory too:

The Einstein action is in fact readily quantized, using gaugefixing and ghost fields ala Feynman, DeWitt, Faddeev, Popov. The background field method used by Hooft and Veltman is most beautiful in this context because it allows one to retain the symmetries of general relativity in the background field, while still gauge-fixing the quantum fluctuations.

This method has, unfortunately, some problems, because the usual nice gauge conditions do not choose only one element out of every class of equivalent solutions-this problem is knowns as "Gribov copies". Moreover, it is conceptually sufficiently complicate, so to use it would be in contradiction with our claim that quantization of gravity is straightforward and essentially trivial. Fortunately, it is not necessary at all, because a much simpler and straightforward method to handle symmetries of a classical theory is sufficient-to break them. Is quantum gravity obliged to have the same symmetry as classical GR? Not at all. All we need is that the Einstein equations can be recovered in some classical limit. Can this limit include the requirement that some symmetry-breaking term has to vanish? Of course. Conceptually this is unproblematic too. Classical theory is some approximation of quantum theory, and approximations quite typically ignore some really existing differences in the more fundamental theory and can have, therefore, larger symmetry groups. So we can, much easier, break the symmetry of the theory by introducing some gauge conditions. And, because one wants to preserve the nice and important fact that the theory as a Lagrange formalism, one would prefer to do this by adding a gauge-breaking term to the Lagrangian. That this modifies the equations of the theory slightly does not matter-all we need is that we can recover the Einstein equations in some limit.

What would be a possible reasonable choice? In GR, we would have to break the general covariance of the theory, by introducing preferred coordinates with a coordinate condition. In GR, there are not many pretenders for a coordinate condition which make sense as a physical equations too. Essentially there is only the harmonic condition. This condition has two important forms - as a condition for the metric field, if it is written in harmonic coordinates, which has the form of a conservation law $\partial_{\mu}\left(g^{\mu \nu} \sqrt{-g}\right)=0$, and as an equation for the preferred coordinates (which will be denoted with $X^{\mu}$ ), namely the harmonic equation $\square X^{\mu}(x)=0$. Given that this is the equation for a scalar field too, one can obtain this equation simply by adding the usual term for a scalar field for each of the preferred coordinates. So we obtain the modified Lagrangian:

$$
L=L_{G R}+L_{\text {matter }}+c_{\alpha} g^{\mu v} \sqrt{-g} \partial_{\mu} X^{\alpha} \partial_{v} X^{\alpha}
$$

which depends on the preferred coordinates, thus, is no longer covariant. With covariance gone away, we have a classical field theory with the usual translational symmetry $X^{\mu}(x) \rightarrow X^{\mu}(x)+$ const (the Lagrangian depends only on derivatives of the $X^{\mu}$ ). And the Noether theorem gives, as usual for such field theories, local energy and momentum conservation laws. So, with such a radical breaking of diffeomorphism symmetry we have also solved the GR problems with local energy and momentum conservation, without any energymomentum pseudo-tensor.

Breaking diffeomorphism invariance leads to additional degrees of freedom for the gravitational field: different choices of the coordinates would now correspond to different physical states, which are distinguished by these additional degrees of freedom. Their character can be easily seen from the Lagrangian: Small modifications of the coordinates would behave like four massless scalar fields, which do not interact in any way with any matter fields.

To summarize: Explicitly breaking diffeomorphism symmetry solves all conceptual problems caused by this symmetry, in particular the problems with the definition of local energy and momentum densities for the gravitational field-something we need if we want to use canonical quantization of a field theory. It is not problematic for the basic requirement that we have to obtain the Einstein equations of GR in some limit.

\section{How to handle causal loops}

What about causality? How to handle causality in a world of quantized general relativity, where causality is defined by the light cone of the metric, but the metric fluctuates because of quantum uncertainty? How one handles in quantum gravity solutions with causal loops? These are questions which seem highly problematic for quantum gravity. So, let's see how they can be handled in our straightforward approach. In unmodified classical GR, we have solution with causal loops, and how to make sense of them given the grandfather paradox remains unclear. But, however uncomfortable these solutions are for GR, there is nothing in GR which would allow to get rid of them.

The field-theoretic approach automatically gets rid of a quite large class of such solutions with causal loops, namely all those with nontrivial topology-what is named "wormholes". But this does not solve the problem with causal loops, because there are solutions with causal loops also on a flat background, with the Gödel universe as the classical example. Moreover, nothing excludes that for initially causally unproblematic solutions causal loops appear later.

But the background introduced by the field-theoretic approach can also be used to define an independent, classical notion of causality. We subdivide the background, which is initially simply an affine space 
$\chi_{0}^{0}$, into space and time, by defining one of the preferred coordinates $\chi^{0}$, as the preferred time $T=\chi^{0}$. Such a subdivision we have to introduce anyway if we want to use canonical quantization. From the point of view of this background, the flow of time is defined by the preferred time $T$. What would be with trajectories which seem in causal-loop-like solutions, to go some time into the past? They would have to be interpreted as the corresponding anti-bodies moving forward in time. A causal loop is, then, nothing more strange than a particle-antiparticle pair creation later followed by the annihilation of the same pair. To use an affine spacetime introduced as a background also for the purpose to define the meaning of space, time, and causality is also a straightforward choice, and solves immediately all similar conceptual questions about the uncertain, quantum nature of space, time and causality: they have no such quantum character but are as in canonical quantum theory, predefined and absolute entities.

The consequence of this is that the spacetime interpretation has to be abandoned. Space and time are defined by the background, not the gravitational field. The gravitational field described, instead, what is measured with clocks and rulers-which do not measure absolute time or distances in absolute space, but something distorted by the gravitational field. And the gravitational field plays a role similar to the old Lorentz ether - a medium which distorts clocks and rulers in such a way that it becomes impossible to measure absolute distances, time, and velocities.

\section{Another way to handle causal loops}

Once something similar to the Lorentz ether appears anyway as the natural interpretation of the gravitational field in quantum gravity, it makes sense to look at the harmonic equations-which have the form of four conservation laws: $\partial_{\mu}\left(g^{\mu \nu} \sqrt{-g}\right)=0$. Why not interpret them as continuity and Euler equations of a Lorentz ether? This was proposed as the General Lorentz Ether in. ${ }^{3}$

The interpretation of the equation $\partial_{t}\left(g^{00} \sqrt{-g}\right)+\partial_{i}\left(g^{0 i} \sqrt{-g}\right)$ as a continuity equation $\rho(x)>0$ leads to another way to handle causal loops. The point is that this interpretation makes sense only if $\rho(x)>0$ everywhere. But that means $\left(g^{00} \sqrt{-g}\right)>0$ everywhere, thus, , has to be a global time-like coordinate. So, no causal loops are possible, because a solution with a causal loop does not have a global time-like coordinate.

One could argue that in this case the existence of GR solutions with causal loops means that such an ether interpretation is not possible. But from point of view of the Lorentz ether, the situation looks different. Usual condensed matter can tear into parts. There is no reason to think that this is not possible for the Lorentz ether, at least in principle. But if this is possible, it means that to cover these situations, the equations near the border of the ether have to be defined. So, the region where the ether density becomes zero simply defines where the condensed matter equations for the ether have reached their boundary of applicability.

This would already be a more serious modification of the theory - in the region where the ether density is zero, no limit allows to recover the Einstein equations. But so what? Where this happens, we have to expect new physics. Do we have to recover the Einstein equations everywhere, or only in the domain of physics where we have observational evidence?

Let's note here also that such an ether interpretation would suggest also another form of a lattice regularization: A co-moving lattice. In such a co-moving lattice, the density would be simply defined by the number of lattice nodes in a given volume. The continuity equation would become a tautology, because the number of nodes remains constant by construction. Moreover, the density would have to be nonnegative by construction too. But this variant - which would have a physical interpretation as an "atomic Lorentz ether" - would have the same qualitative features as the straightforward lattice, namely have only a finite number of degrees of freedom.

For the quantization, the requirement of positivity of $g^{00} \sqrt{-g}$ may be relevant, because $g^{00} \sqrt{-g}$ defines the energy density. The harmonic conditions are essentially the Noether conservation laws. To obtain an energy-momentum tensor in the usual form $g^{\mu v} \sqrt{-g}$, all one has to do is to add to $g^{\mu v} \sqrt{-g}$ a zero in form of the equations of motion. As in GR, they have the form $G^{\mu v} \rightarrow \tilde{G}^{\mu v}\left(g^{\mu v}, X^{\alpha}\right)$. The additional term in the Lagrangian does not modify the matter part, but adds only something to the gravitational part $G^{\mu v} \rightarrow \tilde{G}^{\mu v}\left(g^{\mu v}, X^{\alpha}\right)$. Adding zero does not change anything, so that $g^{00} \sqrt{-g}>0$ leads to $T_{\text {grav }}^{00}+T_{\text {matter }}^{00}>0$ too, so that the energy density is positive everywhere. The possibility of a Lorentz ether interpretation of metric theories of gravity in harmonic coordinates is, different from all the things considered above, not well-known. But all one needs to ensure that the energy density is positive is the requirement that the preferred time coordinate of the background is a global time-like coordinate. And this is a quite natural and often used condition for a time coordinate, so that it can count also as a well-known straightforward thing. The equivalence of this natural condition for the time coordinate and the positivity of the energy density is a nice consequence of using harmonic coordinates.

\section{How to handle non-renormalizability}

The resulting field theory is non-renormalizable. In the past, this was considered to be a death penalty for a field theory - it would be unable to predict anything at all, because one would need an infinite number of constants to define it. But today, based on the Wilsonian approach to renormalization, we know better: All what follows from the theory being non-renormalizable is that it is only an effective field theory. And GR works well as an effective field theory-a large distance approximation of some other, different theory to be used below a critical distance. Here is how Donoghue ${ }^{4}$ summarizes the results:

I. a consistent quantum theory of general relativity exists at energies well below the Planck mass, and that it is necessarily of the form that we call effective field theory.

II. It is common to hear that gravity is different from all our other theories because gravity and quantum mechanics do not go together, that there is no quantum theory of gravity. This is not really the case, as there is no conflict between gravity and quantum mechanics at low energy.

III. While gravity at low energies has a somewhat different structure than other theories, it is not that a quantum theory does not exist. Rather the more accurate statement is that the quantum theory of gravity reveals itself as an effective field theory at low energies and signals that we need a more elaborate theory at high energies.

But what about a theory which works also for in all situations, instead of working only for low energies? The effective field theory approach does not aim to provide such a theory. But in fact to construct such a 
theory is even easier than what has been done developing this effective field theory approach. In fact, the effective field theory approach gives much more-it tells us that all theories of quantum gravity which have the Einstein equations of GR as their classical limit have to give the same results for low energies. But all we need is to construct a single theory of quantum gravity which gives the Einstein equations in some limit. What matters is that it is a well-defined quantum theory, without any singularities and infinities.

But this is easy, and there are standard techniques to do this known as regularization. Regularization is what one has to do to find out that GR is non-renormalizable. Regularization is the method to get rid of the infinities of a field theory, to allow to make computations. Some are quite artificial, and do not care at all about the regularized theory making any sense as a physical theory, like dimensional regularization. But there are other regularizations, which give some well-defined theories without any infinities. The conceptually most simple and straightforward one is lattice regularization. The simplest way would be to replace continuous space $\mathbb{R}^{3}$ by a discrete lattice. If one wants to get rid only of ultraviolet problems, an infinite lattice $\mathbb{Z}^{3}$ would do the job, but one can also restrict oneself to a finite lattice $\mathbb{Z}_{N}^{3}$ with periodic boundary conditions, which could model a large cube. This would remove also all infrared problems from the theory. The fields are defined now on a discrete and finite background, so that the whole theory has only a finite number of degrees of freedom. The qualitative properties of the original theory-Lagrange and Hamilton formalism can be easily taken over from the continuous field to the lattice theory: Instead of the complete field $\varphi(x, y, z)$ we have now only a finite number of field degrees of freedom $q_{i j k}=\varphi\left(x_{i}, y_{j}, z_{k}\right), i, j, k \in\{1, N\}$, as we are used to in canonical quantum theory.

What we need here is only that if the distance between lattice nodes $\lambda$ is small enough, the resulting discrete theory gives approximately the same results as the continuous theory. It should be noted that there are some known problems with lattice approximations which may prevent this. The most serious one is known as fermion doubling. ${ }^{5,6}$ This problem really endangers the viability of a naive lattice discretization-it appears that in the large distance limit of such a naive discretization of the Dirac equation where appears not only one fermion, as described by the original equation, but 16 Dirac fermions. With a more reasonable discretization, so called staggered fermions, the problem can be reduced to four fermions. But if we, in our theory, use only a discretization in space, but not in time, the problem further reduces to two Dirac fermions, and this is already no longer problematic, because in nature (in the standard model) fermions appear only in electroweak pairs. So, fermion doubling does not prevent to model even the standard model of particle physics. ${ }^{7}$ For gravity, there is no similar problem.

The other problem often discussed-chiral lattice gauge theory is instead, irrelevant for the question considered here: It is the problem how to preserve exact gauge symmetries in a lattice theory. Remarkably, there exists a possibility for preserving exact gauge theory even on a lattice, defined by Wilson ${ }^{8}$ But this works only for vector gauge fields. For chiral gauge fields, the gauge symmetry on the lattice will be broken. But, first of all exact gauge symmetry is what one expects for massless gauge fields, and massless gauge fields are vector gauge fields. So, there is no reason to expect exact gauge symmetry for chiral gauge fields. And anyway in our case, the relevant symmetry-diffeomorphism symmetry-has been already been explicitly broken before.
We conclude that lattice regularization defines a viable way to define a theory with a finite number of degrees of freedom, which is, as a finite-dimensional lattice theory, not plagued by the various infinities typical for infinite-dimensional quantum field theories. So, the infinities of QFT do not prevent us, even in principle, from constructing finite, well-defined quantum theories which, in the large distance limit, give the original field theory. Of course, the problem of renormalizability is circumvented here in a quite brutal way. We simply take what is usually considered to be only a worthless intermediate tool-the regularized theory-as the final theory. So, we simply omit all what usually follows-the consideration of a whole series of such regularizations, with various cutoff distances $\lambda \rightarrow 0$, and the attempts to construct a meaningful field theory in the limit $\lambda \rightarrow 0$ . Once we do not even attempt to construct this limit, we do not even have a problem of renormalizability, because this is a problem of the limiting procedure. But each particular theory in this sequence is already a well-defined theory it itself, and those with small enough lattice distance $\lambda$ will be viable, without any necessity to construct the limit of this sequence of theories.

\section{And finally we apply canonical quantization}

It remains to apply finally canonical quantization. Everything is nicely prepared for this: We have, as the background, an absolute space, and absolute time. The breaking of diffeomorphism invariance has given us a non-degenerated Lagrange formalism where the Noether theorem gives us local energy and momentum conservation laws. This is all we need to derive, in the standard way, a Hamilton formalism, which is also non-degenerated. The condition that the preferred time is time-like makes the energy density positive. The lattice regularization does, then, reduce the field theory to a lattice theory with a finite number of degrees of freedom. To quantize such a theory in a canonical way is completely unproblematic.

To summarize, the simple straightforward way to quantize gravity consists of the following steps:

We consider gravity as a field theory on a background spacetime $\mathbb{R}^{3} \times \mathbb{R}$, which describes absolute space, absolute time and causality.

I. We connect it with the gravitational field by explicitly breaking diffeomorphism invariance by adding a non-covariant term to the Lagrangian, which gives the harmonic coordinate condition as an additional equation of the theory. Then we compute the local energy and momentum conservation laws using the Noether theorem, and obtain a non-degenerated Hamilton formalism in the usual way.

II. We add the requirement that the background time coordinate $\mathbb{R}^{\text {. }}$ has to be a global time-like coordinate. This has the consequence that the energy density will be always positive.

III. We regularize the resulting theory using a lattice regularization. The resulting theory has a finite number of degrees of freedom-a lattice on a big cube with periodic boundary conditions.

\section{We quantize the resulting theory using canonical quantization.}

This gives a quantum theory of gravity, which is mathematically well-defined and completely canonical as a quantum theory. In the classical limit it gives a minor modification of GR. This classical limit is not exactly the same theory as GR, because it is defined on $\mathbb{R}^{4}$, thus, has no solutions with nontrivial topology. Moreover, it does not have solutions with causal loops. Nonetheless, in all regions where we have been able to test GR, this theory would give, in the limit where 
the diffeomorphism-symmetry breaking term goes to zero, $c_{\alpha} \rightarrow 0$ ) exactly the Einstein equations of GR. So, from an empirical point of view it is viable, and at least actually empirically indistinguishable from any other imaginable quantization of GR.

All the steps used here to define the theory of quantum gravity are well-known standard techniques, even simplified variants of them. We conclude that constructing a viable theory of quantum gravity is not only not an open problem, but, instead, quite trivial.

\section{It remains to discuss metaphysics}

Without much doubt, the reader will object that this trivial, artificial, nasty construction, which destroys completely all the beauty, all the essential properties of GR, cannot be the True Theory of Quantum Gravity. This would be fine. Because this reaction would support the point of the title-that quantum gravity is not a physical, but a metaphysical problem. Else, the rejection would have to be of the form "nice try, but this theory will be unable to explain observation $\mathrm{X}$ ". It cannot have this form, because all the gravity observation are sufficiently nice explained by the Einstein equations of classical GR, in a domain where one can, without problems, define a space $\mathbb{R}^{3} \times \mathbb{R}$ , and introduce there global harmonic coordinates with one of them being a global time-like one. It cannot have the form of an objection against the mathematical consistency of the theory too, because the whole construction is mathematically sufficiently simple, much simpler than standard QFT. In particular, what is quantized is a theory with a finite number of degrees of freedom.

So, what remains as a reason for rejection? Metaphysics. If the reaction of the reader is a "so what?", I have to admit that this is what I have tried to provoke. I argue already a long time that metaphysics is an important part of physics, and that the positivist rejection of metaphysics is not only invalid, but extremely harmful for fundamental physics. So, in the remaining part of the paper, the questions considered are metaphysical, methodological, and philosophical ones.

\section{A remark about the role of physics of quantum gravity}

Let's start with a methodological point. What makes the difference between a purely metaphysical discussion, and a metaphysical discussion on the background of physics? The metaphysical concepts discussed in physics have to be compatible with physics, which means that they have to be compatible with the actually best existing physical theories, or at least some interpretations of these theories.

So, given that GR and QT are actually these best theories, metaphysical concepts have to be compatible with some viable variants of both. Or, even better, with some compatible quantum theory of gravity. Does it, in this case, matter how ugly the quantum theory of gravity is? No. If one metaphysical concept can present a compatible theory of quantum gravity, and the other not, then the former is compatible with modern physics, and the other not.

Those who like the latter may tend to reject the proposed theory of gravity as ugly. But this does not matter as long as no other, better, more beautiful theory of gravity is presented. Until this happens, this is a serious problem for for the latter metaphysical concept. It is incompatible with modern physics. And it is not a problem of modern physics that it is incompatible with some metaphysical principles, however nice and beautiful these principles are. So, once we have a theory of quantum gravity, which is internally consistent and viable, there is no longer any physical problem. There is only a problem of all those metaphysical ideas and concepts which are not compatible with this theory.

It is completely legitimate for the proponents of these metaphysical concepts to try to develop a competing theory of quantum gravity, which is also well-defined and viable, which is compatible with their own philosophical preferences. What is not legitimate is to reject a well-defined viable physical theory simply because it does not fit their philosophical beliefs. However ugly, it exists. And once the existence and viability of the theory is beyond doubt, the problem is no longer a physical problem, but a problem of those metaphysical concepts which appear incompatible with the known existing theories. So, the existence of at least some viable quantum theory of gravity, however ugly it may be metaphysically, matters in metaphysical considerations about quantum gravity.

\section{A valid metaphysical objections: Missing explanatory power}

With this point in mind, it is worth to note that there exist quite reasonable metaphysical arguments. And such arguments can become decisive if we have to decide between different otherwise viable physical theories or interpretations. If there are no such competitors, they play another important role-they guide the search for improvements of the theory.

One quite strong argument against the construction presented above is insufficient explanatory power. ${ }^{1}$ The theory, by construction, destroys the diffeomorphism symmetry of GR. If the fundamental theory does not have a symmetry which, then, appears in some approximation, this requires explanation: Why is there a symmetry in all the effects we are able to observe if it is not a fundamental symmetry of nature itself? The construction presented above does not suggest anything which would allow to solve this problem. ${ }^{1}$

Here, the variant of this approach based on the Lorentz ether has advantages. The Lagrangian of the Lorentz ether is derived in $^{3}$ from some axioms for the Lorentz ether. The basic point is that the continuity and Euler equations for the ether have to be Noether conservation laws, and these are identified with the Euler-Lagrange equations for the preferred coordinates. Then, given that the equation for the preferred coordinates do not depend on matter fields, the equations for the matter field will also not depend on the preferred coordinates-a consequence of the action equals reaction principle of the Lagrange formalism. But this is already the Einstein equivalence principle. What remains is that the part which depends on the preferred coordinates does not depend on derivatives of the metric. Which gives it, similar to Einstein's cosmological constant, a cosmological character. ${ }^{2}$

But, according to the principles above, this advantage counts only if there is a similar viable quantum theory of gravity for the Lorentz ether too. Fortunately, for a theory with a conservation law there exists

\footnotetext{
${ }^{1}$ Some part of the explanatory power can be considered as part of the physics - the post-dictions (or after-the-fact "predictions") of a theory differ from physical predictions only by the historical order. But different choices of axioms of the theory do not change the physical content of the theory, but can heavily change its explanatory power. So, at least in part explanatory power is a metaphysical property.

${ }^{2}$ Let's note here that the Lorentz-ether-like interpretation of the Standard Model of particle physics in 11 adds even much more explanatory power. Starting from a quite simple model of a lattice of cells, many properties (the gauge group, the number of fermions and all their charges) have been derived there.
} 
also a quite natural lattice regularization with a co-moving lattice. The ether density is, there, simply the density of the nodes of the lattice, and the continuity equation becomes a tautology of the discretization. The most important qualitative property remains unchanged - the number of degrees of freedom is, if the theory is restricted to a cube with periodic boundary conditions, finite. Such a co-moving lattice has also a straightforward physical interpretation as an atomic ether theory, with the lattice nodes as the atoms of the ether.

So, we see that reasonable metaphysical arguments against the simple theory exist, and may appear helpful to improve it. But it would not be sufficient to reject the theory itself as not viable. On the other hand, these possibilities to explain relativistic symmetry are not widely known. If one takes this incomplete state of knowledge about progress reached by outsiders as a fact, the missing explanation of relativistic symmetry remains a valid metaphysical argument. And, even if metaphysical, it is sufficient to explain why many researchers will have a strong inhibitions to break diffeomorphism symmetrythey are simply aware that a final theory without this symmetry would have the obligation to explain the symmetry in the region which we observe.

The strength of this attachment to relativistic symmetry can be observed in discussions of violations of Bell's inequality. Here, we have a similar straightforward solution-the acceptance of a preferred frame, and return to the Lorentz ether, which would allow, in this preferred frame, superluminal causal influences without endangering classical causality. But this is not the preference of the majority. Instead, they favor a rejection of realism as well as causality to preserve relativistic symmetry.

\section{Are there other objections?}

While many researchers of quantum gravity know, at least roughly, the techniques described above, this straightforward approach is not even rejected-it is ignored without presenting any arguments. One can hardly discuss the arguments never given. The few places where such possibilities are mentioned do not give much. Say, there is a short remark about Donoghue's paper ${ }^{4}$ made by Rovelli ${ }^{9}$, in a section named "Old hopes $\rightarrow$ approximate theories":

A small but intriguing group of papers has recently appeared, based on the proposal of treating this perturbative theory seriously, as a respectable low energy effective theory by its own. This cannot solve the deep problem of understanding the world in general relativistic quantum terms. But it can still be used for studying quantum properties of spacetime in some regimes. This view has been advocated in a convincing way by John Donoghue, who has developed effective field theory methods for extracting physics from non renormalizable quantum GR.

Fine, but there is even nothing to argue about. To continue the discussion of the metaphysics, all we can do is to consider metaphysical positions popular in the scientific community-which is also not easy, given the positivist prohibition to discuss metaphysics-and to see if these metaphysical concepts are in conflict with the straightforward approach.

\section{The dream of something fundamentally new about space and time}

The general situation Rovelli ${ }^{10}$ describes in the following words:

In spite of their empirical success, GR and QM offer a schizophrenic and confused understanding of the physical world. The conceptual foundations of classical GR are contradicted by QM and the conceptual foundation of conventional QFT are contradicted by GR. Fundamental physics is today in a peculiar phase of deep conceptual confusion.

Nonetheless, there are aspects where agreement exists. Butterfield and Isham ${ }^{11}$ see wide describe a wide consent about the following:

Despite the variety of programmes, and of controversies, in quantum gravity, most workers would agree on the following, admittedly very general, diagnosis of what is at the root of most of the conceptual problems of quantum gravity. Namely: general relativity is not just a theory of the gravitational field-in an appropriate sense, it is also a theory of spacetime itself; and hence a theory of quantum gravity must have something to say about the quantum nature of space and time.

From this point of view, the field-theoretic approach to GR inherently fails. Yes, it tells something about spacetime. But what it tells about spacetime is the most old news imaginable in modern physics-it is the same what Newton has already written in his Principia. The GR spacetime influenced by matter vanishes completely, gravity is quantized in the most non-relativistic way imaginable. Space and time have no quantum nature at all, they remain fixed and classical.

It seems, the point is not that scientists do not see that the concepts of space and time of quantum theory are very different from those of GR. They see the difference. Quantum theory uses the classical, Newtonian concept of space and time. It even strengthens some of its aspects. So time is explicitly unobservable, every clock will go, with some probability, sometimes even backward in time. ${ }^{12}$ And QFT is nothing but standard quantum theory applied to fields. All what has been reached to make the foundations more "relativistic" is that one has learned what one should not talk about - like the Schrödinger representation - to avoid open conflicts.

But this does not mean that both concepts of space and time-GR vs. QT-are considered as being on equal foot, with quantum gravity being the place where one has to find out which of them is better. The consensus is, clearly, that the concept of space and time of QT has to be given up - because in QT there is no such thing as a "quantum nature" of space and time.

\section{Relationalism as the philosophy of the spacetime interpretation}

At least one part of the quantum gravity research communitythose around loop quantum gravity-has clear and understandable metaphysical reasons to reject this approach. In particular Rovelli gives a clear picture of the metaphysics behind the GR spacetime interpretation. In particular, he sees the origins of the GR philosophy in the much older philosophy of relationalism, supported by Aristotle, Descartes and Leibniz, against Newtonian substantivalism of absolute space and time:

GR changes the notion of spacetime in physics in the sense of relationalism. In pre-relativistic physics, spacetime is a fixed nondynamical entity, in which physics happens. It is a sort of structured container which is the home of the world. In relativistic physics, there is nothing of the sort. There are only interacting fields and particles. The only notion of localization which is present in the theory is relative: dynamical objects can be localized only with respect to one another. This is the notion of space defended by Aristotle and 
Descartes, against which Newton wrote the initial part of Principia. ${ }^{13}$

Rovelli underscores the fundamental, revolutionary character of this philosophy:

GR is much more than just the theory of a specific physical force. Indeed, GR is a theory of space and time. It has modified in depth our understanding of what space and time are, radically changing the Newtonian picture. This modification of the basic physical picture of the world does not refer to the gravitational interaction alone. Rather, it affects any physical theory. Indeed, GR has taught us that the action of all physical systems must be generally covariant, not just the action of the gravitational field. Thus, GR is a theory with a universal reach, whose implications involve the redefinition of our description of the whole of fundamental physics.

The physical picture of the world provided by GR is not that of physical objects and fields over a spatiotemporal stage. Rather, it is a more subtle picture of interacting entities (fields and particles) for which spatiotemporal coincidences only, and not spacetime localization, have physical significance. Once again, this modification of the meaning of the coordinates does not refer to the gravitational force alone: it refers to our entire description of the world at the fundamental level. ${ }^{14}$

For Rovelli, the problem of quantum gravity is that of an "unfinished revolution-it was successful only in the domain of GR, not yet in the quantum world. Quantum gravity has to finish this revolution, and to give a completely relational quantum theory.

Instead, the straightforward field-theoretic approach works in the opposite direction, and returns physics into an substantivalistic, Newtonian picture. In other words, Rovelli's "unfinished revolution" would be completely reverted by an absolutist counterrevolution. So, one would not wonder that a revolutionary like Rovelli does not really like the straightforward approach.

\section{Other effects influencing the metaphysical discussion}

So, we have identified the preference for the relational philosophy of space and time in tradition of Leibniz and Descartes against the Newtonian philosophy of absolute space and time as a key metaphysical conflict. This conflict motivates a rejection of quantum theories of gravity based on the field-theoretic way, which introduces some fixed background spacetime.

Unfortunately there are also a lot of other, metaphysical as well as historical, institutional, sociological and psychological problems which influence and distort modern fundamental physics. Let's shortly mention them.

Job insecurity leading to conformism: In the actual world, scientists have one of the most insecure jobs at all. The typical young scientist has jobs for periods of one or two years. What is standard everywhere else - that you can continue to work if nothing bad happens - is in science a rare privilege of a few "tenured" professors. All other scientist know that their grant is finished in short time, and that they have to look after this for a new job, worldwide, with heavy competition. The basic rule to survive this competition is simple: Publish or perish.

This clearly favors those directions with most grants, most journals to publish, most conferences with conference proceedings. If you work there, there are more jobs to apply, and more possibilities to publish make it more likely to succeed to publish. The predictable result is conformism with the latest fads of the mainstream. The consequence is quite fatal: In fundamental physics, where one would have to expect hundreds of different approaches, given the highly speculative character of the research, we have, in reality, an extreme concentration: There is string theory, there is loop quantum gravity, and everything else is of minor importance.

\section{Novelty as a requirement for success}

Successful scientists are those who find something new. This is quite natural. But it also plays a part in the ignorance of the straightforward approach to quantum gravity considered above. Once all the parts used there are old, well-known things, what would be the base to claim novelty? The positivist prohibition to discuss metaphysics further increases the problem. While presenting new arguments for old philosophical disputes is quite appropriate in philosophy, physicists are restricted here. If there is no novelty about experimental predictions - and there are none in fundamental physics - all what remains are new mathematical techniques or new concepts. None of this is required in the approach considered here.

\section{The physicists today are fascinated by the revolution in physics of the last century}

A triviality, it seems, but this has consequences. If you are not fascinated by the relativistic and quantum revolution, but find classical physics much more impressive, given its ability to give a sufficiently simple, easy to understand picture of the world, which you miss today, will you become a physicist today? Hardly. And this aspect will be even more relevant for those who choose fundamental physics as their domain of interest, instead of experimental physics or domains where the foundations are already well established, like condensed matter theory. But what was one of the main points of this revolution, and one which has a strong potential fascinate young people and to motivate them to become physicists? It is certainly not the ability to compute the correct precession of the Mercury perihelion. What fascinates is the refutation of many things which seemed indisputable to classical physics and to common sense.And so one can expect that they will tend to favor more of it, will favor even more refutations of classical common sense, and will be prejudiced against attempts to recover it.

The danger of mysticism: Modern physics is heavily mathematical. So, a modern physicist also needs a positive relation to mathematics. Mathematics is fascinating in itself - even without applications in physics. A fascination with exceptional, beautiful mathematics, if not confined by common sense, can easily morph into mysticism. This may be especially dangerous in string theory, given that it is based on some really fascinating parts of mathematics.

One of the most fascinating things in mathematics are symmetries. And the more strange a symmetry, the more fascinating it is, increasing the probability of its mystification. Should we wonder why in the discussion of Bell's theorem scientists are ready to give up realism as well as causality, only to preserve the status of Lorentz symmetry as being fundamental?

The positivist inhibition of metaphysical discussions as unphysical: Last but not least, the old positivist prejudice against any metaphysical discussion continues to harm fundamental physics. It has lost some of its power during the last years. In particular, to discuss various interpretations of quantum theory is today much easier. But alternative interpretations of the equations of GR, in particular the Lorentz ether interpretation, remain anathema even today.

As a philosophy of science, positivism is dead since 1935, when 
Popper published his logic of scientific discovery. But the positivist tradition remains surprisingly influential, in particular because the founding fathers of the revolution in physics have been heavily influenced by it, and their ideas, inclusive their misguided positivist ideas, remain the base of teaching modern physics.

\section{Conclusion}

We have seen that the problem of quantum gravity is neither a problem of physics nor of mathematics. The methods of standard, non-relativistic quantum field theory are completely sufficient to construct well-defined canonical quantum theories of gravity, which are completely viable, giving the classical Einstein equations of GR in some well-defined limit. One may not like this approach, for many reasons-but all imaginable reasons are metaphysical in character. So, the problem of quantization of gravity is a metaphysical problem. And, as a metaphysical problem, it is a problem only for those metaphysical concepts which are incompatible with the constructed examples of theories of quantum gravity.

Unfortunately, metaphysical discussions in modern fundamental physics appear to be highly problematic, for a large variety of reasons. We have nonetheless identified as the main metaphysical conflict which distorts quantum gravity discussions the conflict between Newtonian substantivalism of absolute space and time and the relationalism in the tradition of Leibniz and Descartes, which is the base of the spacetime interpretation of GR.

The almost trivial way to quantize gravity we have presented here is based on a Newtonian absolute space and time and the principles of canonical quantum theory. The construction proves that this philosophy is compatible with modern physics. To show that relationalism is compatible with modern physics is, instead, an open problem for the proponents of this philosophy.

It is not a problem of modern physics. The physical problem of quantization of gravity is a trivial one. This triviality has not been recognized up to now based on metaphysical reasons, especially the preference for a particular interpretation of GR.

\section{Acknowledgements}

None.

\section{Conflicts of interest}

Author declares there is no conflict of interest.

\section{References}

1. Oriti D. Approaches to Quantum Gravity: Toward a New Understanding of Space, Time and Matter. Cambridge University Press, UK. 2009.

2. Feynman RP. The Role of Gravitation in Physics, Report from the 1957 Chapel Hill Conference. In: Rickles D, editor. Max Planck Research Library, Germany. 1957.

3. Schmelzer I. A generalization of the Lorentz ether to gravity with general-relativistic limit. Advances in Applied Clifford Algebras. 2012;22(1):203-242.

4. Donoghue J. The Quantum Theory of General Relativity at Low Energies. Cornell University Library, USA. 1996.

5. Gupta R. Introduction to lattice QCD. Cornell University Library, USA. 1998.

6. Chandrasekharan S, Wiese UJ. An Introduction to chiral symmetry on the lattice. Progress in Particle and Nuclear Physics. 2004;53(2):373418 .

7. Schmelzer I. A Condensed Matter Interpretation of SM Fermions and Gauge Fields. Foundations of Physics. 2009;39(1):73-107.

8. Wilson K. Confinement of quarks. Physical Review D. 1974;10(8):24452459 .

9. Rovelli C. Strings, loops and others: a critical survey of the present approaches to quantum gravity. Plenary lecture on quantum gravity at the GR15 conference, Pune, India, 1998. p. 1-26.

10. Rovelli C. Unfinished Revolution. Cornell University Library, USA. 2009.

11. Butterfield J, Isham CJ. Spacetime and the philosophical challenge of quantum gravity. In: Callender C, editor. Physics meets Philosophy at the Planck Scale, Cambridge University Press, UK. 2000.

12. Unruh WG, Wald RM. Time and the interpretation of quantum gravity. Physical Review D. 1989;40(8):2598-2614.

13. Rovelli C. Quantum gravity. Cambridge University Press, UK. 2004.

14. Rovelli C. The century of the incomplete revolution: searching for general relativistic quantum field theory. Jornal of Mathematical Physics. 2000;41(6):3776-3800. 